\title{
Balance Assessment in Older People Using Inertial Sensors
}

\author{
Maryam Ghahramani, Fazel Naghdy, David Stirling, and Golshah Naghdy \\ School of Electrical, Computer and Telecommunications Engineering, University of Wollongong, Wollongong, \\ Australia \\ Email: \{mg846, fazel, stirling, golshah\}@uow.edu.au
}

Jan Potter

Illawara Health and Medical Research Institute, University of Wollongong, Wollongong, Australia

Email: Jan.Potter@sesiahs.health.nsw.gov.au

\begin{abstract}
People at the age of 65 and above are drastically at the risk of falling. Falls among elderly may occur because of many physical factors such as natural deterioration of motor function or other disease related problems such as stroke, Parkinson's or Alzheimer's disease. The methods reported in the literature to assess the risk of falling in older people are often subjective, qualitative, retrospective and inaccurate. An objective, quantifiable approach to assess the risk of falling in elderly by analysing body movement using inertial sensors is conducted. Initial results of the experimental work and analysis carried out on three subjects, one older person with impaired balance and two younger people with normal balance are reported. The sensors are mounted on the subjects' chests and they are asked to conduct balance tests. The results of the data analysis indicate that the elderly subject has disturbed and involuntary chest movements. This demonstrates the potential of the method to assess balance and the risk of fall in older people.
\end{abstract}

Index Terms-balance assessment for older people, fall risk assessment between the elderly, gait and posture analysis

\section{INTRODUCTION}

It is reported that almost $30 \%$ of people above 65 years of age fall each year all over the world. This figure even increases to $40 \%$ for people older than 80 [1]. Fallers are at risk of further falls and $52 \%$ of them fall again in the following 12 months [2]. Almost $10 \%$ of falls in older people lead to injury such as fractures or head injury [3]. Falls have the highest percentage of injury-related deaths and fall related injuries have the highest cost after vehicle injuries [4], [5]. The mortality rate of fall increases dramatically with age and $70 \%$ of accidental deaths in persons above 75 are caused by falling [6].

A fall is defined as a swift and unintentional change in position leading the person to descend to a lower surface and level such as the ground [7]. A fall can be triggered by two groups of factors; intrinsic caused by inherent physical deficiencies such as visual impairment and postural hypotension, and extrinsic caused by the

Manuscript received December 30th, 2013; revised May 8th, 2014 environment and surroundings conditions such as a slippery floor. The intrinsic factors are associated with the effect of ageing on the body [8], [7].

Many studies have focused on identifying the intrinsic fall factors. Factors such as dizziness caused by medicine taken, loss of balance, syncope, postural instability, visual problems, neurological feebleness, muscle weakness caused by natural deterioration, and sensorimotor deficiencies have been identified as possible intrinsic fall factors [9].

Balance plays a key role in fall prediction. Keeping one's balance, an automatic process in healthy people, can be challenging for the elderly while doing daily chores. An individual with normal balance is capable of reacting to weak perturbations. Balance control changes as a consequence of illness, ageing, and disease. A balance control problem in aged people may be the result of deterioration in vestibular, visual, and somatosensory systems. Moreover the prevalent diseases in the elderly intensify a balance disorder [10].

There are a number of balance tests available for fall risk assessment. A well-known method called the Berg Balance scale consisting of 14 activities, such as sit to stand and standing on one leg, is widely used for fall risk assessment in older people. Participants are scored by a clinician on each task, on a scale of 0-4 and finally all the scores are added to produce a total score out of 56. It is assumed that individuals who can control their balance have better ambulatory ability [11]. As the tests are scored by clinicians visually, the method is prone to human error.

The primary aim of this multidisciplinary study is to identify an effective solution for fall risk assessment and mobility in older people and develop a method to objectively quantify them. In this study we explore whether the balance and risk of a fall in older people can be assessed through the analysis of body posture and movements.

Our primary assumption is that acceleration at thorax level is a prominent factor in fall risk analysis. Body sway in quiet standing is like the motion of an inverted pendulum. According to inverted pendulum model of up- 
right posture it has been proven that centre of pressure (COP) displacement determines the COM acceleration [12] and the total body balance can be achieved by the regulation of the total body COM about the supporting foot [13]. The ankle and hip moments are associated with the joint moments required to move the COP in the direction of the COM displacement. The moment acts to decelerate the chest and trunk, preventing lateral collapse and falling [12].

A pendulum sways to and fro in a quasi-regular mode while balancing and the sway is defined as unidirectional movement between one reversal point and the next [14].

Exactly like a pendulum any instability and movement at the feet level get intensified at the chest level (Fig. 1)

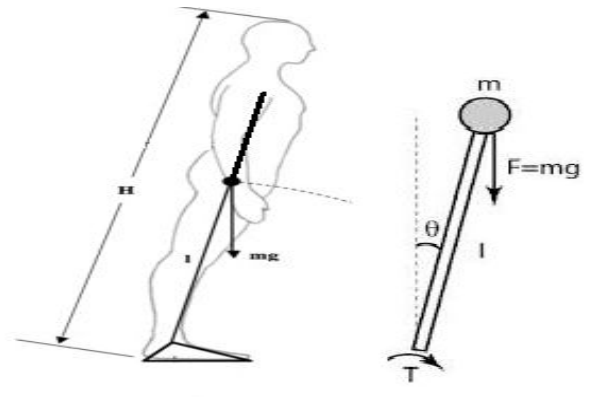

Figure 1. Inverted pendulum model of body

\section{METHOD}

Experimental Set Up: The body mounted inertial sensors can be accelerometers, miniature gyroscopes, magnetometers, pressure sensors or foot switches and goniometeres. More advanced body mounted devices integrate a number of these sensors into one device.

In this project MTw Development Kit from Xsens technology is deployed to measure gait. MTw is a small, highly accurate wireless inertial 3D motion tracker consisting of 6 inertial sensors. MTw has many benefits compared to similar inertial sensors. MTw kit is fully wireless and portable, consequently quite practical and easy to set up and deploy. The kinematic data is initially recorded in MT Manager, a Windows-based software package. The data is subsequently analysed, using an intermediate program coded in MATLAB.

Experimental Design: This study is conducted at University of Wollongong, Australia. The project is approved by the University's Human Research Ethics Committee and all the subjects participating in the experimental work are informed of the project and the nature of their involvement in the experimental work. They are also asked to sign a consent form. The work reported in this paper is based on the experimental work carried out on three subjects. The subject number one is a female at the age of 65 with impaired balance. The second subject is a healthy young woman aged 25 , and the third subject is a healthy young man aged 25 . For the first experiment, subjects are asked to stand still trying not to move at all. The acceleration of chest is recorded using an MTW sensor mounted on their chests. In the first experiment, the candidates are asked to stand fully stationary with eyes open. In the second experiment, the candidates still stand stationary but with eyes closed for one minute. The acceleration of chest along $\mathrm{X}, \mathrm{Y}$ and $\mathrm{Z}$ axes are recorded. A sample of the magnitude of the acceleration data, $\operatorname{acc}(\mathrm{t})$, recorded by MTW is shown in Fig. 2,2 where $\operatorname{acc}(\mathrm{t})=\sqrt{(\operatorname{accX}(\mathrm{t}))^{2}+(\operatorname{accY}(\mathrm{t}))^{2}+(\operatorname{accZ}(\mathrm{t}))^{2}} \quad$ and $\operatorname{acc} X(t), \operatorname{acc} Y(t)$ and $a c c Z(t)$ are the acceleration components along $X, Y$ and $Z$ axes ,respectively.

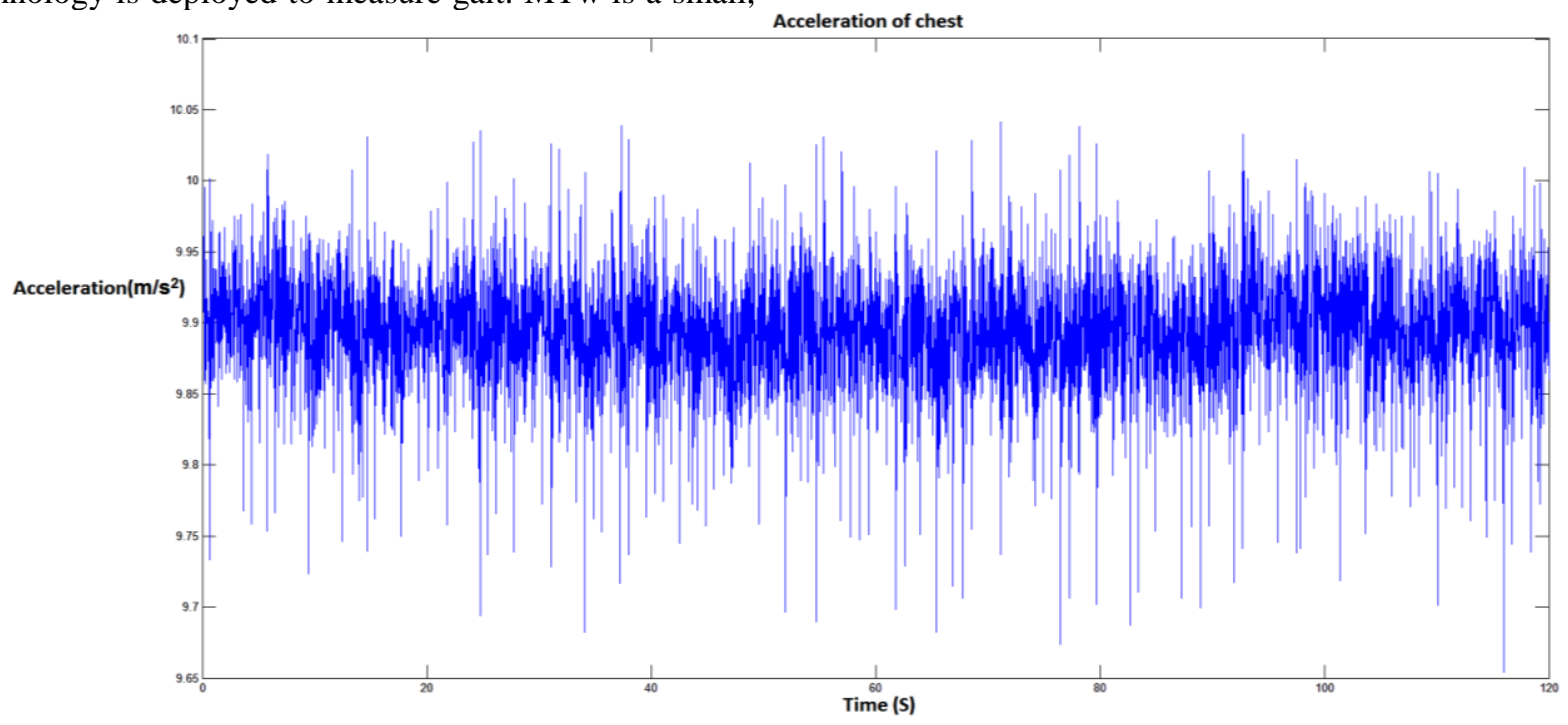

Figure 2. The acceleration of chest

Data Analysis: Due to its high dimensionality, the data captured by inertial sensors is typically aggregated and modelled using various Gaussian Mixture Models (GMM), producing a random number of multivariate Gaussian distributions. Each distribution has a specific mean and variance [15]. In this process, the multidimensional data from different subjects are clustered into a number of statistically different clusters [16]. 
The result of clustering of the chest acceleration data using GMM for the 3 subjects while standing with eyes open and closed using GMM is shown in Fig. 3.

In these diagrams, the acceleration data is grouped into 15 different clusters. For subject 1 shown in Figs. 3a, the chest acceleration with eyes open assumes many clusters which is indicative of significant involuntary movement of the subject to stay stationery. The GMM sequences of the chest acceleration data for the younger subjects are shown in Fig. $3 \mathrm{~b}$ and $3 \mathrm{c}$. It is clear that the motion of the chest for these two subjects are limited to cluster 1, indicating that the younger subjects have much less involuntary movements at chest level.

Fig. 3d, 3e and $3 \mathrm{f}$ consecutively show the results of GMM clustering of the chest acceleration data for the three subjects while standing with eyes closed. The diagrams show that the acceleration data covers more clusters when the eyes are closed. This indicates that all the subjects require more movement to stay stationary and maintain balance with closed eyes.

The histograms of the number of chest acceleration data for each cluster corresponding to each scenario of Fig. 3 are shown in Fig. 4. The histograms show that the data from subject 1 with eyes closed has the highest abundance of the sequences. This can be interpreted as the subject has the most involuntary movement of the chest and these movements increase while she stands with eyes closed. Even the data cluster distribution has changed for the younger subjects while standing with eyes closed comparing to standing with eyes open. This indicates that vision plays a significant role in maintaining balance.
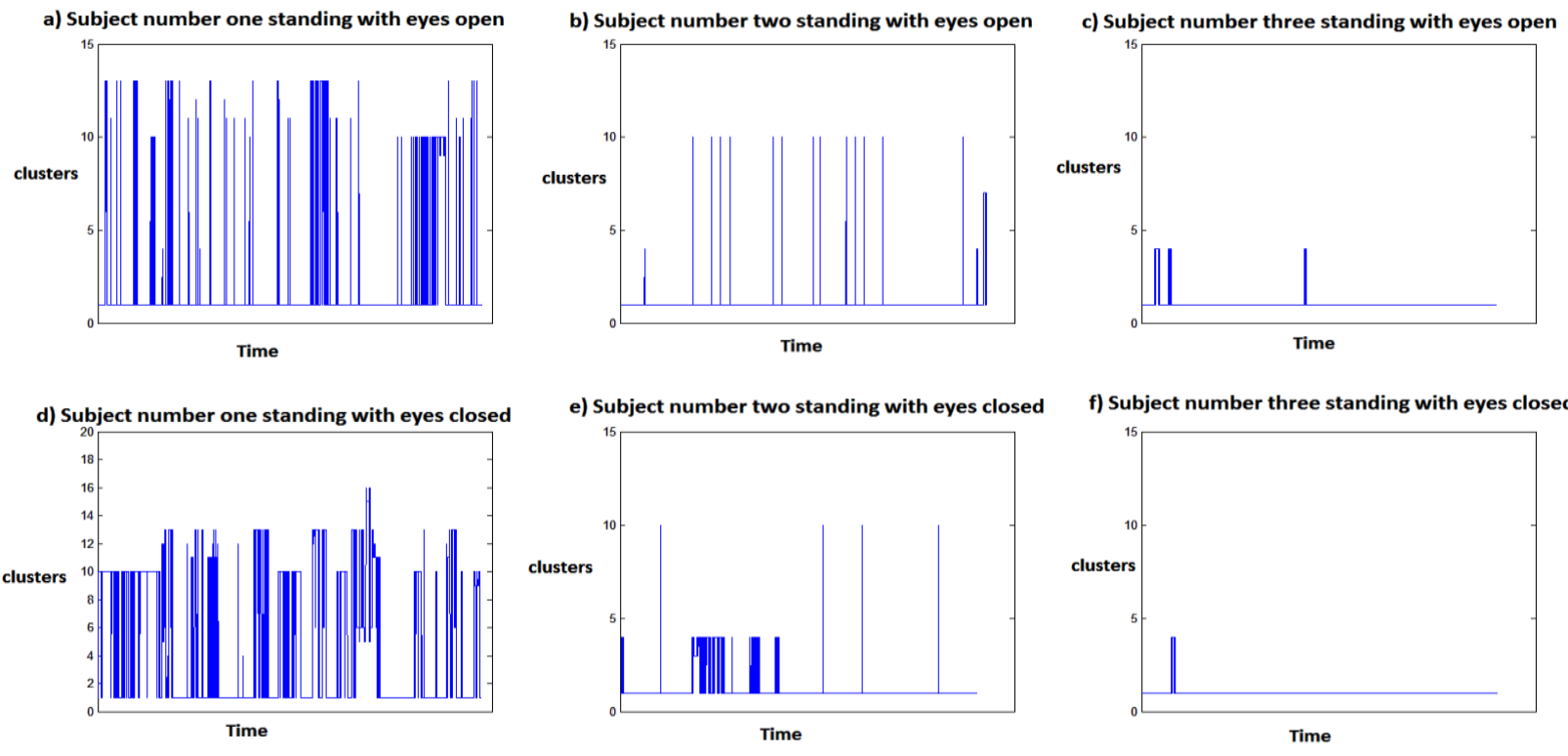

Figure 3. Clustering of the chest acceleration data using GMM for experiments 1 and 2
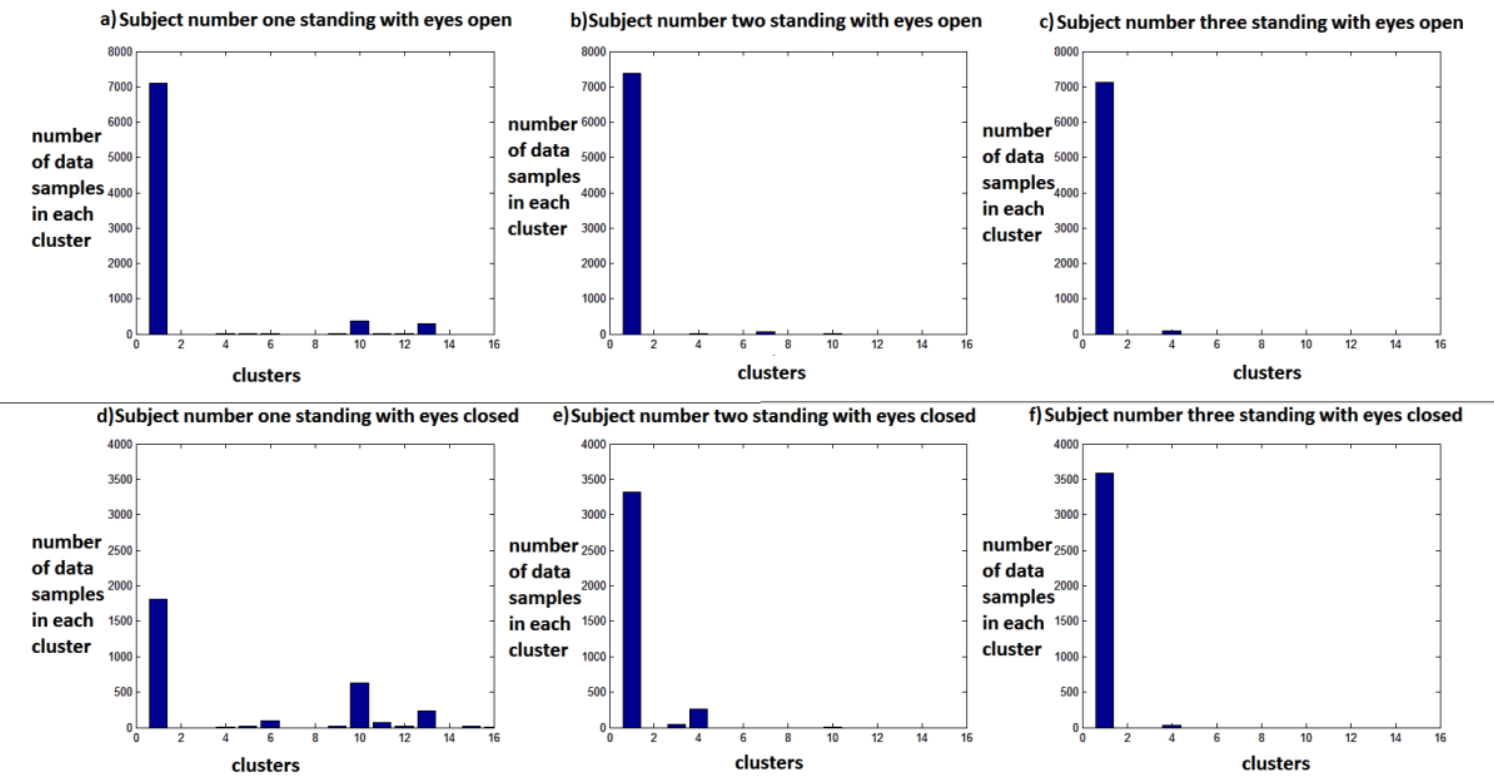

Figure 4. The histogram of the GMM clusters shown in Figure 3 


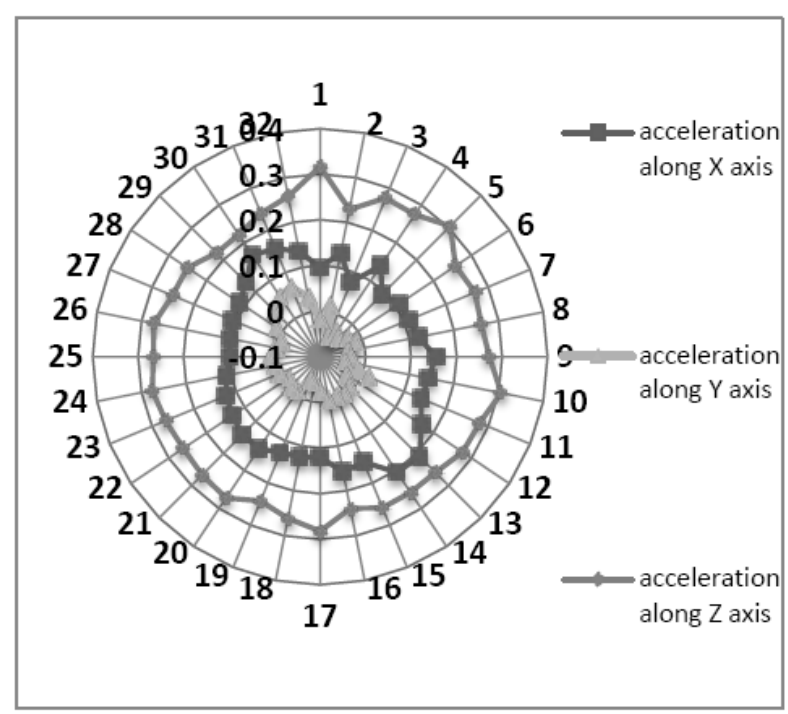

Figure 5. The radar diagram of 3-dimonsional acceleration data fitted in cluster number 4

Each cluster consists of a set of acceleration data with a specific mean and variance. For instance the 3 dimensional chest acceleration data labelled as cluster 4 is shown in Fig. 5. In this radar diagram the acceleration values of data set in cluster number 4 along each axis relative to the centre point are illustrated. The diagram indicates that the data set in this cluster has a special mean value and all the data along each axis has a defined distribution from the centre and has a special variance.

The subjects are also asked to conduct sitting, stand to sit, and standing on one leg tests. In the stand to sit test, the subjects sit down on a chair. The data clustering of the sitting test is shown in Fig. 6. The test has three phases of standing phase; stand to sit phase and sitting phase.

In the sitting test, subjects sit still for 120 seconds with back unsupported and arms folded. The data clustering in Fig. 7 again shows involuntary chest movement for the elderly subject.

The standing on one leg test has three phases of "standing on both legs phase", "bringing one leg up phase" and "standing on one leg phase". In Fig. 8, it can be seen that the elderly subject's balance is disturbed several time during standing on one foot.
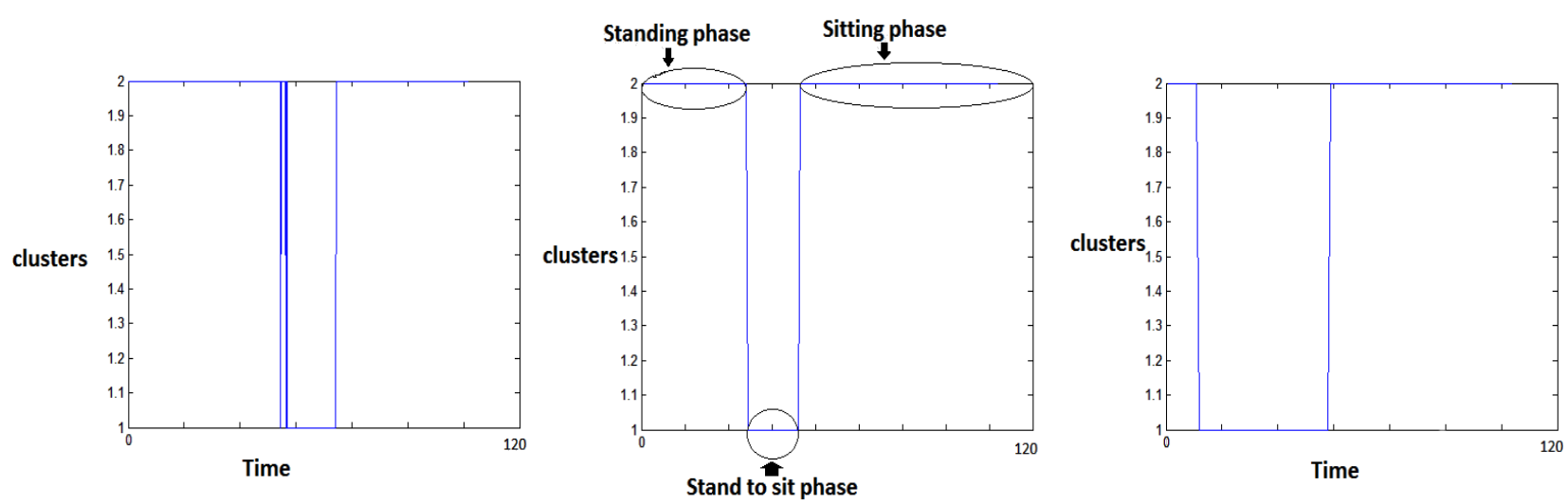

Figure 6. Clustering of the chest acceleration data using GMM for "Stand to Sit" test

a) Subject number one sitting unsupportedly

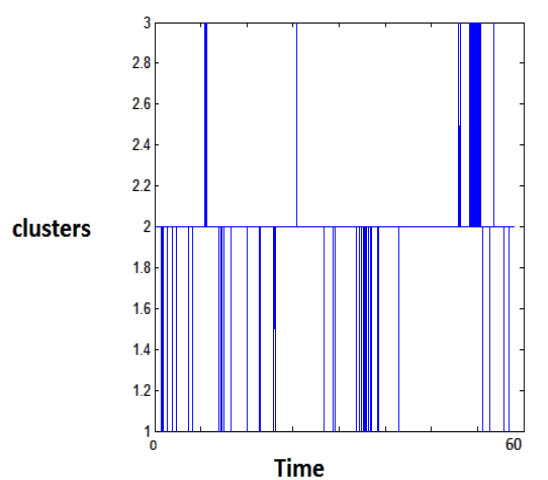

b)Subject number two sitting unsupportedly

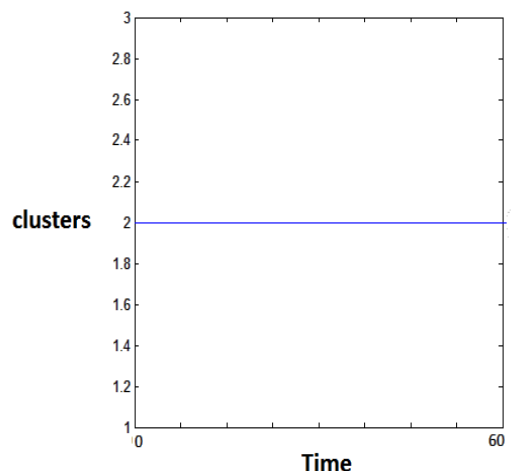

c) Subject number three sitting unsupportedly

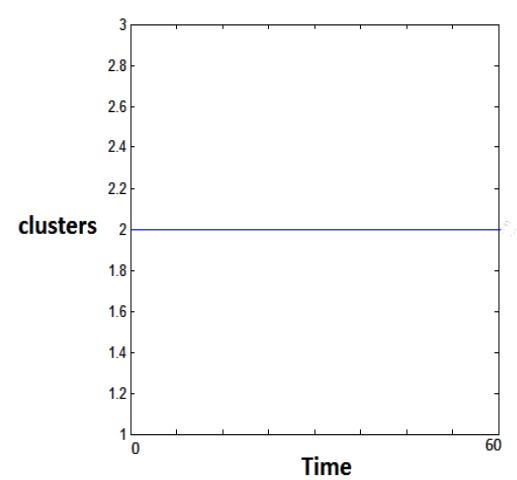

Figure 7. Clustering of the chest acceleration data using GMM for "Sitting" test 
a)Subject number one bringing up one leg and standing on one leg
b)Subject number two bringing up one leg and standing on one leg
c)Subject number three bringing up one

leg and standing on one leg
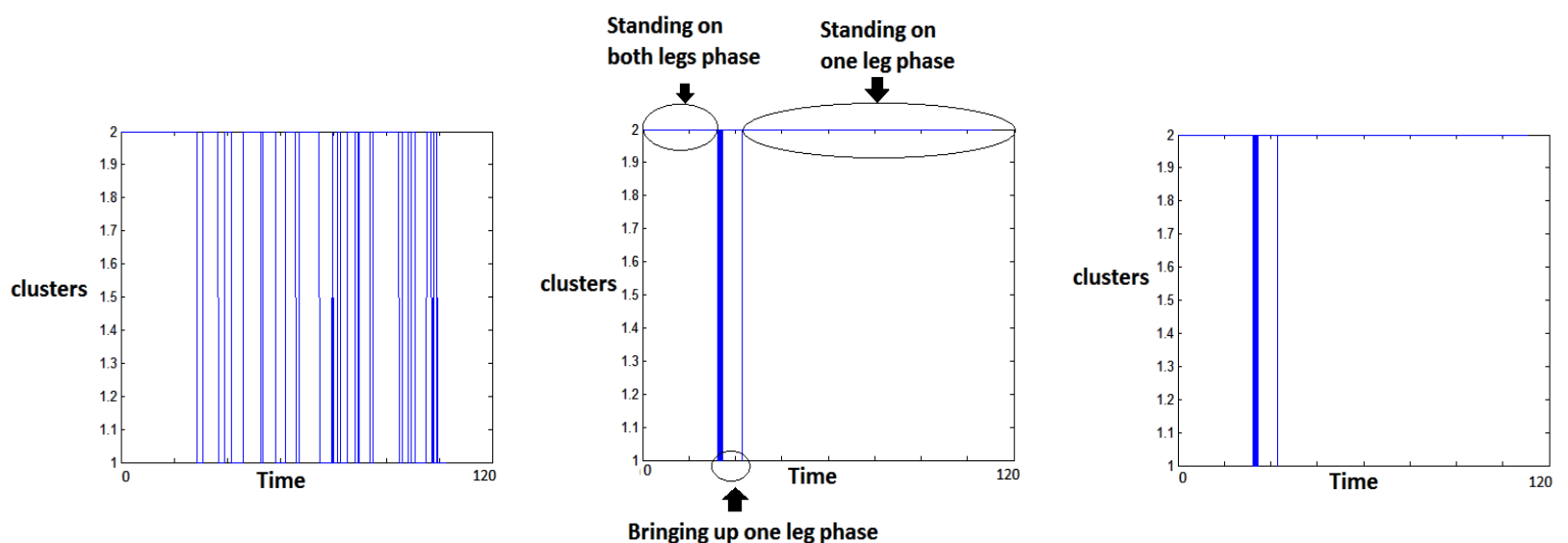

Figure 8. Clustering of the chest acceleration data using GMM for "Bringing up one leg and standing on one leg" test

\section{DISCUSSION}

Our proposed system has the following features, which give novelties to our work.

- Only one sensor at chest level is used and the sensor is so light and unobtrusive. It has no interference with subjects' movements.

- The acceleration of chests of subjects doing common balance tests is analysed. The tests are usually used at clinics and they are scored visually which make them subjective and prone to human error. Our proposed method is completely objective.

- The disturbed balance can easily be distinguished using the GMM data clustering method as presented in Fig. 3, Fig. 6, and Fig. 8.

\section{CONCLUSION}

The early stage of the work conducted to assess the risk of fall in older people using inertial sensors was presented in this paper. The analysis of the acceleration signal obtained from the sensor mounted on the chest provides an indication of the balance control of a subject and according the risk of fall.

Future work will include further experimental work on older people with balance impairment and more extensive analysis of the data to develop an effective indicator of fall.

\section{REFERENCES}

[1] M. Tinetti and C. Williams, "Falls, injuries due to falls, and the risk of admission to a nursing home," The New England Journal of Medicine, vol. 337, pp. 1279-1284, 1997.

[2] J. Close, M. Ellis, R. Hooper, E. Glucksman, S. Jackson, and C. Swift, "Prevention of Falls in the Elderly Trial (PROFET): A randomized controlled trial," Lancet, vol. 353, no. 9147, pp. 93-97, 1999.

[3] M. E. Tinetti, "Preventing falls in the elderly persons," The New England Journal of Medicine, vol. 348, pp. 42-49, 2003.
[4] A. C. Scheffer, N. Van Dijke, and T. Van der Hooft, "Fear of falling: Measurement strategy, prevalence, risk factors and consequences among older persons," Age and Aging, vol. 37, no. 1, pp. 19-24, 2007.

[5] A. Shumway-Cook, S. Brauer, and M. Woollacott, "Predicting the probability for falls in community-dwelling older adults using the timed up \& go test," Physical Therapy, vol. 80, no. 9, pp. 896-903, 2000.

[6] F. B. Vellas, F. Cayla, and J. de Pemille, "Prospective study of restriction of activity in old people after falls," Age and Aging, vol 16, no. 3, pp. 189-193, 1987.

[7] G. Feder, C. Cryer, and S. Donovan, "Guidelines for the prevention of falls in people over 65," British Medical Journal, vol. 321, pp. 1007-1011, 2000.

[8] T. Ikezeo, Y. Asakawa, H. Shima, and N. Ichihashi, "Physical function screening of institutionalized elderly women to predict risk of falling," Japanese Journal of Physical Fitness and Sports Medicine, vol. 58, no. 5, pp. 489-498, 2009.

[9] Y. Lajoie and S. Gallagher, "Predicting falls within the elderly community: comparison of postural sway, reaction time, the berg balance scale and the Activities-specific Balance Confidence (ABC) scale for comparing fallers and non-fallers," Archives of Gerontology and Geriatrics, vol. 38, pp. 11-26, 2004.

[10] K. Berg, "Balance and its measure in the elderly," Canada Phsiotherapy, pp. 240-246, 1989.

[11] T. J. Stevenson, D. M. Connelly, J. M. Murray, D. Huggett, and T. Overend, "Threshold berg balance scale scores for gait-aid use in elderly subjects: A secondary analysis," Physiotherapy Canada, vol. 62, no. 2, pp. 133-140, 2010.

[12] S. Rietdyk, A. Patla, D. Winter, M. Ishac, and C. Little, "Balance recovery from medio-lateral perturbations of the upper body during standing," Journal of Biomechanics, vol. 32, no. 11, pp. $1149-1158,1999$.

[13] C. D. MacKinnon and D. A. Winter, "Control of whole body balance in the frontal plane during human walking," Journal of Biomechanics, vol. 26, no. 6, pp. 633-644, 1993.

[14] I. D. Loram and M. Lakie, "Human balancing of an inverted pendulum: Position control by small, ballistic-like, throw and catch movements," The Journal of Physiology, vol. 540, pp. 11111124, 2002.

[15] W. Press, S. Teukolsky, W. Vetterling, and B. Flannery, "Gaussian mixture models and k-means clusters," in Numerical Recipes: The Art of Scientific Computing, New York, Cambridge University Press, 2007, pp. 842-847.

[16] D. Stirling, F. Naghdy, G. Naghdy, and M. Field, "Objective functional capacity assessment using inertial sensors," in Proc. First IEEE International Conference on Healthcare Informatics, Imaging and Systems Biology, 2011. 


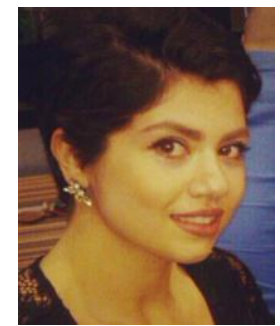

Maryam Ghahramani was born in Iran 1987. She received the B. Sc. Degree in Electrical engineering from Shiraz University, Shiraz, Iran. She is currently working toward her Master of philosophy degree in the area of fall risk analysis between the elderly people at University of Wollongong, Wollongong, Australia.

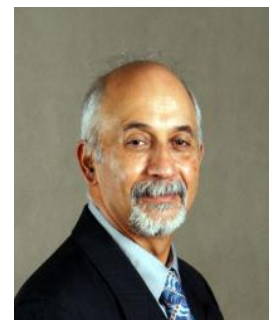

Fazel Naghdy was born in Tehran, Iran. He received his first degree from Tehran University in 1976. He then received an MSc from the Postgraduate School of Control Engineering, University of Bradford, England, in 1980 and received his $\mathrm{PhD}$ from the same University in 1982. Fazel Naghdy has a demonstrated track record and leadership in research, teaching, and management. His research has had its focus on machine intelligence and control particularly in embedded mechatronics and robotics systems. He has more than 270 publications in international journals and conferences and as book chapters. He is also contributing reviewer to IEEE Transactions on Mechatronics Engineering, and International Journal of Intelligent Automation and Soft Computing. He has served on a large number of International scientific committee of various international conferences. He is the Director of Centre for Intelligent Mechatronics Research. His current research interests include haptic rendered virtual manipulation of clinical and mechanical systems, intelligent control and learning in non-linear and nonstructured systems. He is currently a Professor of Robotics and Intelligent Systems at University of Wollongong.

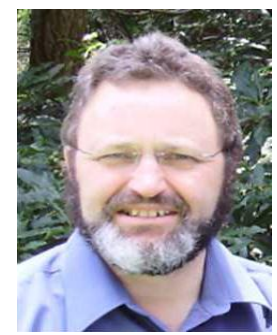

David Stirling obtained his BEng degree from the Tasmanian College of Advanced Education (1976). He further obtained his MSc degree (Digital Techniques) in Digital Techniques from Heriot-Watt University, Scotland UK (1980), and his PhD from the University of Sydney (1995). He has worked for over 18 years in several industries, most recently as a Principal Research Scientist with BHP Steel, where he established over 30
Industrial Machine Learning and Data mining applications during this period. Subsequently, he established and operated for several years a successful consultancy in the data-mining domain. He has recently taken up a position as Senior Lecturer at the University of Wollongong. His research interests are in Machine Learning, Data Mining, Robotics, Autonomous and Complex Systems.

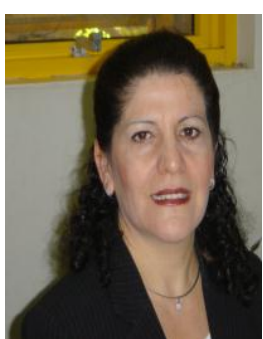

Golshah Naghdy is an Associate Professor at the School of Electrical, Computer and Telecommunication Engineering, University of Wollongong. She received her BSc in Electrical Engineering and Electronic Engineering from Sharif (Aryamehr) University, Tehran. She did her Post-graduate studies in England where she received an MPhil in Control Engineering and PhD in Electrical and Electronic Engineering. The application of her $\mathrm{PhD}$ work in the automation of a wastewater treatment process was subsequently patented in England. Golshah was a Senior Lecturer at Portsmouth University before joining Wollongong University in 1989. Her research interests include biological and machine vision in particular a generic vision system based on "wavelet neurons" and its application in the development of artificial retina implants, medical image processing, content based image retrieval, and robotics.

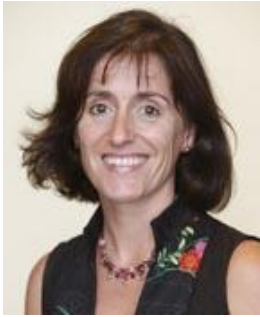

Jan Potter is senior staff specialist at the Acute Geriatric Unit and Stroke Unit of the Wollongong Hospital and the clinical director of the Aged Care, Chronic Care and Community Health stream of the Southern Hospitals Network of the South Eastern Sydney Illawarra Area Health Service. This position covers both the Illawarra and Shoalhaven regions and includes nine hospitals. Clinical Professor Potter has a strong background in medical research and education, with a focus on geriatric care in the United Kingdom (UK) and Australia. In the UK she served for seven years as an honorary senior lecturer in geriatric medicine at the University of Glasgow, and for five years as hospital sub-dean for medicine. Since arriving in Australia in 2004, she has led the development of a comprehensive geriatric service in the Illawarra 